\title{
Feasibility of fast-particle channeling in quasicrystals
}

\author{
T. Kupke and U. Peschke \\ Institut für Theoretische und Angewandte Physik der Universität Stuttgart, D-7000 Stuttgart 80, Germany \\ H.-D. Carstanjen \\ Institut für Theoretische und Angewandte Physik der Universität Stuttgart, D-7000 Stuttgart 80, Germany \\ and Max-Planck-Institut für Metallforschung, Institut für Physik, D-7000 Stuttgart 80, Germany \\ H.-R. Trebin \\ Institut für Theoretische und Angewandte Physik der Universität Stuttgart, D-7000 Stuttgart 80, Germany
}

(Received 15 March 1991)

\begin{abstract}
The feasibility of ion channeling in quasicrystalline structures is investigated in a computer experiment. For this purpose, a quasilattice is constructed by the grid method. As this model crystal shows, also in a quasicrystal the lattice points are arranged in strings and planes and form pronounced axial and planar channels. Their occupation by lattice points is, however, not constant, but exhibits a wide density distribution in contrast to periodic crystals. Manganese atoms were placed at the lattice points and backscattering yield profiles of $750-\mathrm{keV} \mathrm{He}^{+}$ions were obtained in a channeling computer simulation. Pronounced channeling minima are observed in the profiles for axial as well as planar channeling.
\end{abstract}

Up to the present time, about 100 binary and ternary metallic alloys have been synthesized which display noncrystallographic icosahedral symmetry in their electron diffraction patterns and, if grown macroscopically, also in their morphology. Since the icosahedral symmetry contains a fivefold axis, the structures cannot be periodic. Many models have been proposed such as twinned periodic crystals, ${ }^{1}$ randomly stacked oriented icosahedral clusters of atoms (the icosahedral glass ${ }^{2}$ ), and the threedimensional icosahedral Ammann tiling of space ${ }^{3}$ (as a generalization of the two-dimensional Penrose pattern ${ }^{4}$ ). The Ammann tiling, which is the cell structure for a large class of the so-called quasicrystals, fills space in a nonperiodic way with two types of rhombohedra, a prolate and an oblate one (Fig. 1), whose edges are parallel to the center-vertex vectors of an icosahedron and which are stacked according to well-defined matching rules. ${ }^{5}$ Relaxation of the rules leads to random tilings, which at higher temperature might be stabilized due to the increase of the entropic part of the free energy $F=U-T S{ }^{6,7}$ For complete structural information the positions of the atoms within the Ammann rhombohedra must also be given. "Decorations" of the basic tiles with atoms were proposed by a comparison with related crystalline compounds ${ }^{8}$ and on the basis of neutron-scattering data with contrast variations. ${ }^{9}$ In the latter experiments, atoms with differing neutron-scattering cross sections were substituted, so that partial structure factors could be determined. These were evaluated by a generalization of the Ammann tiling model, i.e., the method of atomic hypersurfaces, ${ }^{10,11}$ and led to the following result for $\mathrm{Al}_{74} \mathrm{Mn}_{20} \mathrm{Si}_{6}$ : The $\mathrm{Mn}$ atoms occupy the corners of the Ammann rhombohedra with a certain frequency of occurrence. The $\mathrm{Al}$ atoms are placed-also with certain frequencies-at preferred positions of the faces, and the $\mathrm{Si}$ atoms at three different sites along the long diagonal of the prolate rhombohedron.

These data have been obtained only for particular quasicrystalline compounds and have to be confirmed by other methods, as a series of unproved assumptions entered the evaluation. For example, in the contrast measurements it is always taken for granted that the structure remains unchanged when one sort of atoms is substituted by another one.

One method which has been used successfully for the structural analysis of both perfect and defected periodic crystals, but which has not been applied yet to quasicrystals, is ion channeling. For periodic crystals it has been proven first by computer simulations ${ }^{12}$ that properly aligned atomic rows and planes can guide ions deeply into the crystal, thus reducing the ion-backscattering rate appreciably. Soon afterwards the simulation was confirmed by experiment. ${ }^{13-15}$ Before attempting the same experiment in quasicrystals, it is advisable to investigate
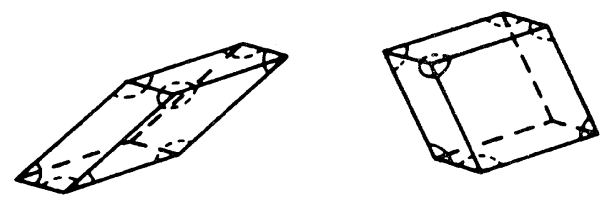

FIG. 1. The two elementary cells of a quasicrystalline structure: a prolate (thin) and an oblate (thick) rhombohedron. Angles of faces: $63.435^{\circ}$ (full lines) and $116.565^{\circ}$ (dashed lines). 
their column and layer structure and to calculate channeling backscattering profiles by suitable simulation procedures.

For this purpose a slab of a primitively decorated icosahedral Ammann lattice of dimensions $20 a \times 20 a \times 134 a$ was constructed on a computer according to the grid method. ${ }^{16,17}$ The edge length $a$ of the basic rhombohedra (Fig. 1) was taken $a=0.46 \mathrm{~nm}$ as for the Mn-Mn separation in Al-Mn-Si. The $z$ coordinate was placed along a fivefold symmetry axis, the $y$ coordinate along a twofold axis. Then the string and layer structure of the system was analyzed. Figure 2 shows the projection of the lattice points along the $z$ axis. The 84113 lattice points project into only 1408 points in the plane, which implies that they are aligned in rows. As concerns channeling the most important properties of the structure are as follows:

(1) The lattice points are aligned in well-defined strings. Each dot in Fig. 2 is the shadow of up to 100 points (on the average 60). The projection pattern is quasiperiodic with locally fivefold symmetry.

(2) The strings form distinct axial channels, but of various shapes, predominantly pentagonal prisms.

(3) The occupation of the strings with lattice points (the occupation density) is not constant, rather displays a wide distribution. In Fig. 2, the occupation density of the various strings is indicated by circles of different radius. The distribution is illustrated further in the histogram of Fig. 3. It exhibits two broad maxima, at about 0.7 and 1.1 lattice points per $\mathrm{nm}$.

(4) From Fig. 2, also the existence of well-defined planes becomes evident. In Fig. 4, the lattice points of each plane encountered along the path $A-A^{\prime}$ of Fig. 2, are projected to the $y$ axis, and the density of vertices in each $x-z$ plane is plotted versus its $y$ coordinate. The planes form a quasiperiodic sequence with three different separations of ratios $1: \tau: \tau^{2}$, where $\tau$ is the golden number $(1+\sqrt{5}) / 2$. The occupation densities fluctuate by about a factor of 10; however, if the densities of adjacent

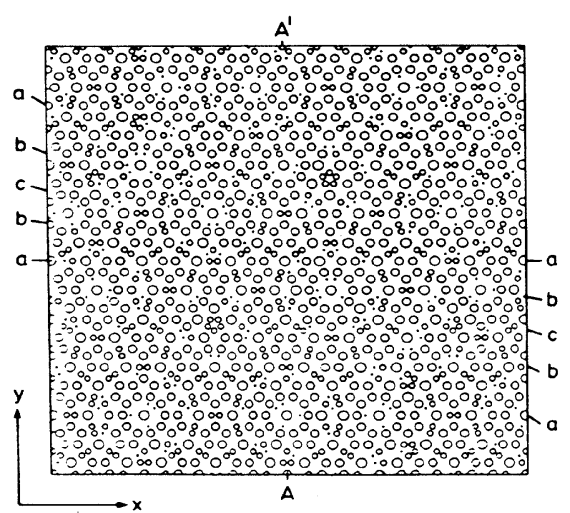

FIG. 2. Projections of the vertices of the model quasicrystal (see text) along the fivefold $z$ axis. Each circle corresponds to up to 100 (on the average 60) vertices. These are arranged in pronounced strings and planes forming axial and planar channels. The number of vertices per string is, however, not constant; this is indicated by circles of different radius.

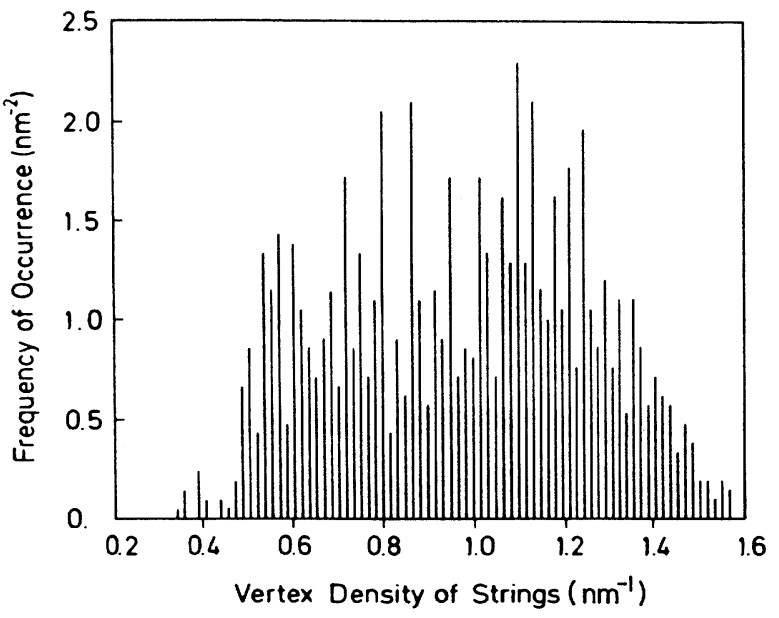

FIG. 3. Distribution of the density of vertices per string in the model quasicrystal. It exhibits two maxima at about 0.7 and 1.1 lattice points per $\mathrm{nm}$, respectively; the average occupation density amounts to 60 vertices per string.

planes with smallest separation are added, the distribution is almost constant.

Using this model quasicrystal, ion channeling along the fivefold $(z)$ axis was simulated on a computer. Ionbackscattering yield profiles were calculated for angular scans across the axial channel of fivefold symmetry andwith a tilt plane $6^{\circ}$ off the $y$ - $z$ plane-across various planar channels containing this axis.

The principles of such calculations may be found described in detail in Refs. 18 and 19. For the present case of ion channeling and backscattering by a quasicrystal, they will now be summarized.

A set of ions of equal momenta, and hence equal energies and angles of incidence, is impinging with uniform spatial distribution on the surface of the quasicrystalline

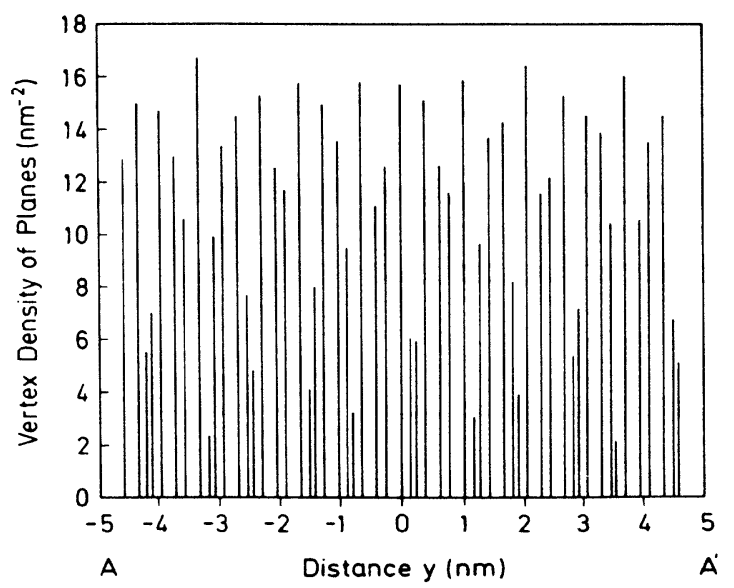

FIG. 4. Planar arrangement of vertices and occupation densities as encountered along a path $A-A^{\prime}$ indicated in Fig. 2. There exist only three different distances between neighboring planes which are related as $1: \tau: \tau^{2}[\tau=(1+\sqrt{5}) / 2=$ golden number]. 


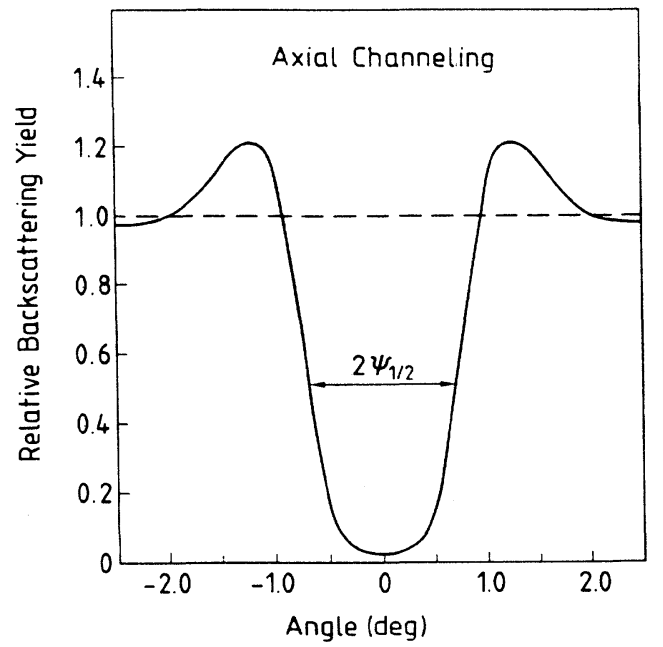

FIG. 5. Backscattering yield profiles of $750-\mathrm{keV} \mathrm{He}^{+}$ions as obtained from the channeling computer simulation of an angular scan across the fivefold axis of the model quasicrystal (Mn atoms placed at the vertices of an Ammann quasilattice). The dip proves that ion channeling works also in quasicrystals; the angular half-width amounts to $\psi_{1 / 2}=0.687^{\circ}$.

structure. To simulate a finite beam collimation, normally distributed fluctuations of the momenta are allowed for. The ion trajectories are calculated considering only binary collisions with the lattice atoms. Only collisions with ion-atom distances less than $20 a_{\text {TF }}$ (the Thomas-Fermi screening radius) are taken into account. Scattering angles are calculated in momentum approximation. The ion-atom interaction was assumed to be given by the Thomas-Fermi-Moliere potential.

The backscattering rate was obtained by adding up in every single collision the probability $P\left(s_{i j}\right)$ for an ion $j$, which passes a lattice point $i$ at distance $s_{i j}$, to hit the thermally vibrating atom $i$. With $\bar{u}$ being the linear rms vibrational amplitude of the atom, $P(s)$ reads

$$
P(s)=\left(1 / 2 \pi \bar{u}^{2}\right) \exp \left(-s^{2} / 2 \bar{u}^{2}\right) .
$$

The calculations simulated channeling of $750-\mathrm{keV} \mathrm{He}^{+}$ ions by a quasicrystal which consisted of $\mathrm{Mn}$ atoms placed at the vertices of the quasilattice. This decoration is a first approximation to a real structure such as Al-Mn-Si. Apart from the basic length $a=0.46 \mathrm{~nm}$ of the rhombohedra the rms amplitude $\bar{u}$ of the Mn atoms was taken as $7 \mathrm{pm}$. The trajectories of $5000 \mathrm{He}^{+}$ions were followed up to a penetration depth of $134 a$ for various sets of angles of incidence. In order to shorten the computer time electronic and nuclear stopping of the ions was neglected as well as multiple scattering by the crystal electrons. However, these are not serious restrictions, because the ion penetration depths are very shallow.

Figures 5 and 6 show two of the relative backscattering rates versus the angle of ion incidence $\psi$. The profiles have been normalized to unity at large off-channel angles. The profile for channeling along the icosahedral axis (Fig. 5) exhibits a pronounced minimum for perfect

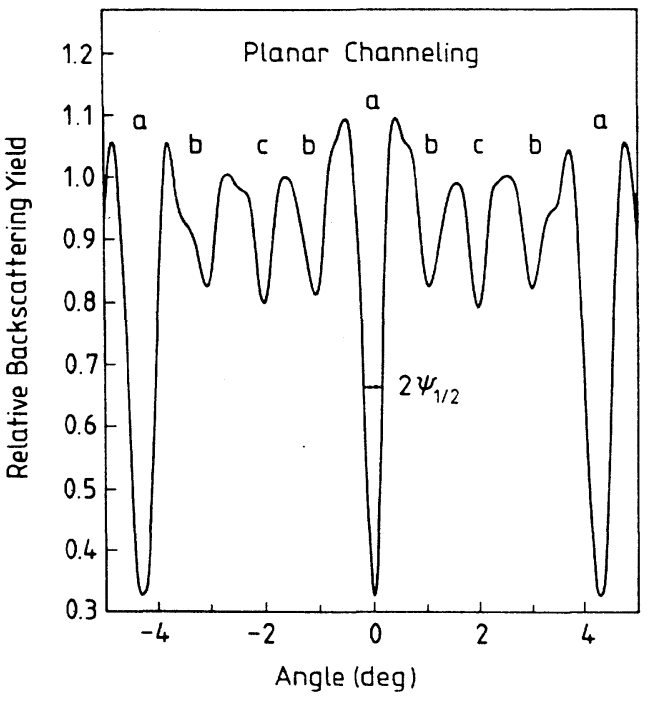

FIG. 6. Ion-backscattering yield profile as obtained from an angular scan (plane of angular tilt $6^{\circ}$ off the icosahedral axis) through the main planar channel system of the model quasicrystal. The dips marked by $a$ are due to channeling in these planes. Besides, smaller dips (marked by $b$ and $c$ ) are observed which are due to channeling in minor planar channels. Their orientations are indicated in Fig. 2 by the same letters.

alignment of the ion beam with the crystal axis $(\psi=0)$ with a half-width (HWHM) of $\psi_{1 / 2}=0.687^{\circ}$. Roughly the same halfwidth is obtained by employing Barrett's formula ${ }^{18}$ originally derived for channeling in periodic crystals:

$$
{ }_{B} \psi_{1 / 2}=0.8 F_{\mathrm{RS}}\left(1.2 \bar{u} / a_{\mathrm{TF}}\right) \psi_{1}
$$

Here $\psi_{1}=\left(2 Z_{1} Z_{2} / d E\right)^{1 / 2}$ is the critical angle for axial channeling according to Lindhard, ${ }^{20} F_{\mathrm{RS}}$ is a function related to the interaction potential of the incident ions and an atomic string, $\bar{u}$ is the rms thermal amplitude of the lattice atoms, $a_{\mathrm{TF}}$ the Thomas-Fermi screening radius, $E$ is the ion energy, and $Z_{1}, Z_{2}$ are the atomic numbers of the incident ions and the lattice atoms, respectively. The structural information for a crystal is expressed in Barrett's formula by the interatomic distance $d$ of the atoms in a row. If for the quasicrystal the average interatomic distance $\bar{d}=1.019 \mathrm{~nm}$ of the strings is inserted, one arrives at ${ }_{B} \psi_{1 / 2}=0.626^{\circ}$, about $9 \%$ less than the result of the computer simulation.

Figure 6 shows the backscattering yield profile for an angular scan through the major planar systems marked by $a$ in Fig. 2. Besides three wide dips (at $\psi=0^{\circ}$ and $\psi= \pm 4.2^{\circ}$ ) which are due to these major planar channels and correspond to a tenfold symmetry of the channeling pattern, narrower dips are also observed (indicated by $b$ and $c$ in Fig. 6). They are caused by less densely occupied minor planar channels (for instance, $c$ planes are halfway planes, i.e., at $18^{\circ}$ between planes $a$ ) which are hardly visible in Fig. 2 and are therefore indicated by marks. Furthermore for channeling by the major pla- 
nar system, reasonable agreement is found between the angular half-width obtained in the computer experiment $\left(\psi_{1 / 2}=0.174^{\circ}\right)$ and the value of ${ }_{B} \psi_{1 / 2}=0.160^{\circ}$ from Barrett's formula for periodic crystals:

$$
\begin{aligned}
& { }_{B} \psi_{1 / 2}=0.72 F_{\mathrm{PS}}\left(1.6 \bar{u} / a_{\mathrm{TF}}, d_{P} / a_{\mathrm{TF}}\right) \psi_{P} \\
& \psi_{P}=\left(2 \pi n_{P} Z_{1} Z_{2} e^{2} a_{\mathrm{TF}} / E\right)^{1 / 2}
\end{aligned}
$$

Here $F_{\mathrm{PS}}$ is a function related to the interaction potential between ions and atomic planes. Again, the structural information for the sample is the interplanar distance $d_{P}$, which is related to the occupation density of the planes by $n_{P}=N d_{P}$ ( $N$ is the volume density of atoms, here $N=16.44 \mathrm{~nm}^{-3}$ ). Again an average, namely $\bar{n}_{p}=2,69$ $\mathrm{nm}^{-2}$, has been employed. Similarly to axial channeling, ${ }_{B} \psi_{1 / 2}$ is about $8 \%$ larger than $\psi_{1 / 2}$.

These differences are significantly larger than expected from the statistical errors of the computer simulation (for periodic structures, our computer code reproduces Barrett's critical angles ${ }_{B} \psi_{1 / 2}$ within $1 \%$ ). Very likely they are due to the distribution of occupation densities of strings and planes encountered in a quasicrystalline structure. An analytical treatment of channeling for structures of nonconstant occupation densities, which could yield insight into these interrelations, is in progress.

The Mn-decorated model quasicrystal investigated may be considered as skeleton for an Al-Mn-Si quasicrys- tal. When this skeleton is filled with $\mathrm{Al}$ and $\mathrm{Si}$ atoms, we expect that part of them will line up with the strings and planes formed by the $\mathrm{Mn}$ atoms, thus reinforcing the channeling properties but barely changing the backscattering dips of Figs. 5 and 6 . Another part will form additional strings and planes which-due to the low $Z_{2}$ of $\mathrm{Al}$ and $\mathrm{Si}$-will weaken channeling, or-if the occupation density is low-will cause dechanneling. For backscattering by these $\mathrm{Al}$ or $\mathrm{Si}$ atoms one may even obtain completely different structures ${ }^{21}$ which give information on the positions of these atoms in the frame of the Mn quasilattice.

With respect to the analysis of quasicrystals we expect that a real channeling backscattering experiment can provide information about (i) the string and planar structure and, hence, symmetry of the quasicrystal in a direct way, as in contrast to diffraction methods the channeling patterns image the real lattice structure; (ii) the mean occupation density of the strings and planes by atoms and, as related quantities, the mean interatomic distances in strings and mean interplanar distances; (iii) the decoration of the quasicrystalline structure by the various types of atoms encountered in these compounds by a proper analysis of the energy of the backscattered ions. Since in periodic crystals channeling proved a most effective method to spot atoms off regular positions (e.g., between crystal planes), it is most probably sensitive also to disorder in quasicrystals and will be able to discern between deterministic and random tilings.
${ }^{1}$ L. Pauling, Phys. Rev. Lett. 58, 365 (1987).

${ }^{2}$ P. W. Stephen and A. T. Goldman, Phys. Rev. Lett. 56, 1168 (1986).

${ }^{3}$ P. Kramer and R. Neri, Acta Crystallogr. A 40, 580 (1984).

${ }^{4}$ R. Penrose, Bull. Inst. Math. Appl. 10, 266 (1974).

${ }^{5}$ A. Katz, Commun. Math. Phys. 118, 263 (1988).

${ }^{6}$ C. L. Henley, J. Phys. A 21, 1649 (1988).

${ }^{7}$ P. Bancel, Phys. Rev. Lett. 25, 2741 (1989).

${ }^{8}$ C. L. Henley and V. Elser, Philos. Mag. B 53, L59 (1986).

${ }^{9}$ For a review, see Ch. Janot and J. M. Dubois, J. Phys. F 18, 2303 (1988).

${ }^{10}$ D. Frenkel, C. L. Henley, and E. Siggia, Phys. Rev. B 34, 3649 (1986).

${ }^{11}$ P. Bak, Phys. Rev. Lett. 56, 861 (1986).

${ }^{12}$ M. T. Robinson and O. S. Oen, in Proceedings of the Conference Le Bombardement Ionique, edited by J. J. Trillat (CNRS, Paris, 1962); Appl. Phys. Lett. 2, 30 (1963); Phys. Rev. 132, 2385 (1963).
${ }^{13}$ G. R. Piercy, F. Brown, J. A. Davies, and M. McCargo, Phys. Rev. Lett. 10, 399 (1963).

${ }^{14}$ H. Lutz and R. Sizmann, Phys. Lett. 5, 113 (1963).

${ }^{15}$ R. S. Nelson and M. W. Thompson, Philos. Mag. 8, 1677 (1963).

${ }^{16}$ N. G. de Bruijn, Ned. Akad. Weten. Proc. Ser. A 84, 39 (1981); 84, 53 (1981).

${ }^{17}$ D. Levine and P. J. Steinhardt, Phys. Rev. B 34, 596 (1986).

${ }^{18}$ J. H. Barrett, Phys. Rev. B 3, 1527 (1971).

${ }^{19}$ H. D. Carstanjen and R. Sizmann, Radiat. Eff. 12, 211 (1972).

${ }^{20}$ J. Lindhard, K. Dan. Vidensk. Selsk. Mat. Fys. Medd. 34, No. 14 (1965).

${ }^{21}$ For the case of $\mathrm{Ti}$ and $\mathrm{O}$ in $\mathrm{TiO}_{x}$, see G. Della Mea, A. V. Drigo, S. Lo Russo, P. Mazzoldi, S. Yamaguchi, G. G. Bentini, A. Desalvo, and R. Rosa, Phys. Rev. B 10, 1836 (1974). 